\title{
Deep Levels in Semiconductors
}

\author{
P.J. Dean, Malvern
}

(Royal Signals and Radar Establishment)

Introduction to Defect States

Here we are concerned with a particular class of localised energy states, induced in the bandgap of a semiconductor. The host states can be represented by undamped electron wavefunctions. Localised states produce strongly damped wavefunctions characteristic of stationary states located at the point at which the exact translational symmetry of the perfect crystal is broken by the presence of a defect. We may consider a point defect, such as a missing or interstitial host atom or an impurity, or an extended defect, for example a dislocation or a stacking fault. Real crystals usually contain both classes of defects. Point defects are often deliberately introduced to provide some desired electrical characteristic of the semiconductor, typically from electron donating or accepting substitutional impurities. Other defects, again usually impurities, may be introduced to provide desirable optical characteristics, for example luminescence of a particular colour or decay time. The properties provided by these defects depend upon their electronic properties, determined by the way in which their characteristic electronic states interact with those of the host semiconductor. In general, this is an extremely complex matter, involving a multi-electron problem even if the system can be described by a central force at a single atomic site in the crystal lattice.

However, in certain circumstances this complexity becomes resolved in an elegant and simple manner. This is so for those very impurities of the greatest practical significance for the control of electrical pro- perties. The binding energies, $E_{t}$, they exhibit for excess electronic particles must be sufficiently small to permit nearly complete ionisation at typical device operating temperatures, $\geq 300 \mathrm{~K}$. The characteristic kinetic energy $k T$ is then $\geq 25 \mathrm{meV}$, and the condition for strong thermal ionisation means that $E_{\mathrm{t}}$ must be $\leq 0.1 \mathrm{eV}$. The theoretical understanding of such shallow centres is rather straightforward and complete.

\section{General Properties of Deep States}

Truly deep states are the antithesis of the shallow states. They include donor and acceptor states in which the short range potential has surpassed useful treatment by perturbation theory. This occurs when $E$ $\geq 0.1 E_{\mathrm{g}}$, where $E_{\mathrm{g}}$ is the bandgap of the semiconductor. We must also consider neutral centres, such as the so-called isoelectronic traps like $N_{\text {in }}$ GaP. Then, the entire binding is due to the short range difference in the screened potential of the two atoms since the impurity central cell is neutral before the electron is bound. Such neutral traps differ very fundamentally from charged donor or acceptor states. Their binding energies are usually small, perhaps a consequence of local atomic relaxation effects, and may be comparable with the donors or acceptors. However, binding to a short range potential $V$ requires a critical value of the product $V_{\mathrm{o}} a_{\mathrm{c}}^{2}$ as a pre-requisite for the appearance of even a single bound state. Such centres do not possess the infinite series of shallow excited states characteristic of the long-range screened Coulomb potential of a charged centre.
When the wavefunction of the deep state is expanded in terms of the eigenfunctions of the perfect crystal, it is no longer the case that contributions come from a very restricted region of the Brillouin zone in the vicinity of the energetically nearest band edge, as for the shallow centre. In fact, the energy level for a deep state is determined by a relatively delicate balance between conflicting contributions from different regions of the host band structure. This means that properties such as the deformation potentials which characterise the response to elastic strains are quite different from those for shallow states, even when the actual binding energies are not significantly different. The symmetry properties of the deep state in this case govern the matrix elements describing contributions from host states of different symmetries as well as the selection rules for optical transitions to these states.

\section{Experimental Techniques for Deep Levels}

The experimental study of deep levels received considerable impetus through a growing recognition during the 1970 s of their practical importance. For example deep levels conspire to shorten the lifetime of injection lasers and, on a more positive aspect are important in controlling the Fermi level in semi-insulating semiconductors. Shallow levels were mainly studied by electrical methods in the early days of semiconductor physics, but optical techniques soon proved superior for the fine resolutions required to discriminate between nearly effective mass-like states. Optical techniques have also yielded a great deal of information on some deep states, particularly those where lattice relaxation is sufficiently modest to provide structured absorption and luminescence spectra, with

\section{Microcomputer Quantum Mechanics}

\section{J Killingbeck}

The increase in availability of microcomputers means that many students todau know a computer language but have no experience of applying it to scientific problems. This practical text shows how computation can be integrated with theoretical analysis as part of a unified attack on problems in one of the most interesting areas of modern physics.

April 1983 177pp hardcover ISBN 0-85274-455-2 £11.95

\section{Electrostatics 1983}

Sampuran Singh (ed)

Institute of Physics Conference

Series 66

This volume contains four invited and 22 contributed papers, and a further 13 papers presented at two poster sessions. They indicate the present activity in the science and technology of electrostatics, now a growth area of interest as a result of the wide use of plastics with their associated 'static' problems, and the everincreasing technological importance of electrostatic processes and devices.

April 1983 262pp hardcover ISBN 0-85498-157-8 £20.00
New paperback

\section{Parallel Computers} Architecture, Programming and Algorithms

\section{R W Hockney \& C R Jesshope}

A practical introduction to operating supercomputers for students and professional users, describing and comparing five machines in detail. Well written, comprehensive in detail

highly recommended to anyone seeking an introductory text on parallel

processing. Computing Reviews

April 1983 423pp paperback ISBN 0-85274-752-7 £9.95 
good luminescence efficiency. Electrical techniques particularly suited to measure deep levels, especially those near the active region of typical semiconductor devices, involve various forms of junction space charge spectroscopy. The level is detected through its contribution to the junction capacitance or current associated with a change of charge state induced either thermally (deep level transient spectroscopy) or optically (photo-capacitance or photocurrent). These techniques have the advantage that they provide direct access to many parameters of interest, carrier capture cross-sections as well as photoionisation cross-sections, concentrations and of course binding energy. Measurements of the dependence of capture crosssection on temperature and carrier concentration give information on the mechanism of energy loss, radiative, multiphonon or Auger. Variations in defect annealing rates with minority carrier injection level have provided vital evidence of recombination induced defect reaction and diffusion processes. Such processes are of particular significance for the primary intrinsic defects produced by radiation damage. The identification of the particular defect associated with each energy level has proved to be an exacting task, however.

\section{Accuracy Required from Deep Level Theories}

Experimental discrimination between deep states and their practical influence depends upon their exact energy depth to an accuracy of a few tenths of an $\mathrm{eV}$ or less. The scale of atomic energy levels is very much larger, $\sim$ tens of $\mathrm{eV}$. Thus, the factors responsible for the reduction in energy scale which accounts for the appearance of energy levels from many impurities and defects within the 1-2 eV bandgap of a typical semiconductor must be estimated with precision. This means that the strength and form of $V_{0}$ must be wellrepresented at short range, a difficult task. The central cell problem is usually simplified by the use of a pseudopotential, smoothed in the core region to represent the potential difference between an impurity ion and the host atom it replaces. Small changes in the localisation of the electron charge in the central cell region have a very large influence on the level depth. Only a small proportion of the total charge is usually localised in this critical region. For example, electron spin resonance suggests that only $\sim 25 \%$ of the single electron bound to the very deep $\mathrm{P}_{\mathrm{Ga}}$ antisite donor in $\mathrm{GaP}$ resides in the central cell.

\section{Effects of Lattice Relaxation}

Substantial changes in valence electron wavefunction are-likely near a centre with a strong local pseudopotential. The valence electron relaxation thus produced generally alters the lattice stability around the defect, producing new equilibrium lattice co-ordinates. These co-ordinates depend on the charge state of the defect. The charge state changes in many methods of detection of deep states, for example in a photo or thermal ionisation event. The lattice relaxation associated with the change of charge can be very strong. It may be totally symmetric, or contain a symmetry-breaking term as is normally the case when one of the basic electronic states is orbitally degenerate. Then, the Jahn-Teller theorem predicts that this degeneracy will be lifted through a lattice distortion which splits the energy state, leaving the centroid of the substrates unshifted.

\section{Difficulties of Deep Level Theory}

All these effects make theoretical treatments of the properties of deep states very difficult. Often, only one characteristic of the state may be known experimentally, usually the energy depth. Even this may be difficult to derive accurately from experiment in the presence of large lattice relaxation. It is not possible to make an entirely first principles calculation of the energy of a truly deep state to the accuracy really required to distinguish between various levels which may be observed experimentally. Thus, centres are not generally identified through an appeal to pure theoretical treatments. The best that can be obtained is an accuracy of a few tenths on an eV, similar to the accuracy of the determination of host energy states, for example using large scale Green's Function techniques. However, this may be sufficient to distinguish between conflicting models. For example, the ground state symmetry of a defect may be established experimentally, and the theoretical energy separation between states of different symmetry, such as the singlet and triplet energy states formed from combinations of the dangling bonds at a vacancy in a tetrahedrally bonded semiconductor, may be much larger than the uncertainty of either state, as for $V_{\mathrm{Ga}}$ in GaP. When ESR appeared to find an orbital singlet state in the energy gap, the only tenable conclusion was that the centre could not be $V_{\mathrm{Ga}}$. However, more detailed experiments showed that this state was indeed orbitally degenerate, but perhaps with a rapid re-orientation rate between equivalent spontaneous distortions (dynamic Jahn-Teller effect). The experimental results are thus consistent with theoretical expectation for the $V_{\mathrm{Ga}}$ attribution.

\section{Deep Level Theoretical Techniques}

We cannot do justice in this short review to the complex theoretical techniques used to calculate deep state properties. The pseudopotential Slater-Koster Green's function technique works best with a defect of high symmetry and uses a formalism which exploits the basic translational symmetry of the host semiconduc- tor. The changes in properties on introduction of a deep state are emphasised, for example changes in the energy dependence of the density of electron states. Impurity complexes and reconstructed (strongly distorted) centres introduce complications which make the cluster approach more attractive. There, a small part of the crystal surrounding the defect is treated as a "defect molecule". The defect molecule treatment of Hjalmarson et al is an extreme variant of this approach. It is particularly useful for the insight provided into the character of the resonant and gap states, but is mainly of a qualitative nature. The properties of the cluster are usually handled within a molecular orbital framework. The difficulty with this approach is the determination and treatment of an optimum interface between the cluster and the rest of the crystal. The cluster must not be too large so that the molecular orbital calculations remain tractable, yet large enough so that the results are not too sensitive to the properties of the interface and its treatment, for example saturation of the surface orbitals by monovalent atoms. Both techniques involve extensive numerical computations for quantitatively reliable results. Both predict for a very deep donor, a hyper-deep impurity-like state, resonant with the valence band, and a host-like state of similar symmetry within the semiconductor bandgap.

\section{Results of Deep Level Calculations}

Many calculations have been done, for example on the properties of $V_{\mathrm{Si}}$ in Si. Important and interesting properties, such as the negative $U$ behaviour in which the single donor level lies below the second donor level due to Jahn-Teller distortion within the single donor state, are well represented by the Green's function theory. The electronic and vibronic properties of the deep $0_{p}$ donor in $\mathrm{GaP}$ also appear to be well represented by a theory which assumes the usual, strong hybridisation between the $p$ electrons of 0 and the valence electrons of the host lattice. This classical model for a donor in a covalent semiconductor accords well with experiment. However, controversy remains with a quite different description in which this valence hybridisation does not occur. The atomic description is familiar in the properties of transition and particularly rare earth series impurities in solids. The atomic $3 d$ and $4 f$ energy states are more or less strongly perturbed by the relevant local crystal field at the impurity ion, but otherwise uninfluenced qualitatively but only affected quantitatively through the magnitudes of the level splittings. This description works well except for the nearly filled shell species such as $\mathrm{Cu}$.

Recent theoretical studies of Vogl using an extension of the defect molecule approach give a remarkably good account of 


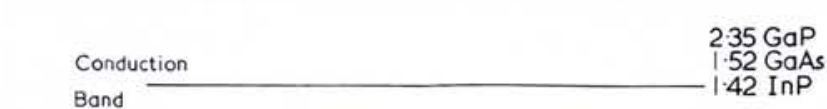

GaAs -

GaP ........

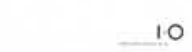

InP

10

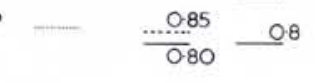

0.75

\begin{tabular}{l}
0.70 \\
0.52 \\
\hline
\end{tabular}

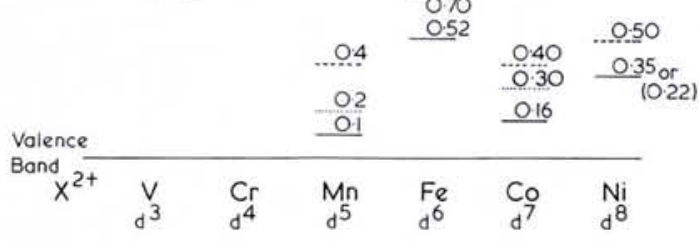

the experimental ground state energies for substitutional transition metals in III-V semiconductors, including those shown in Fig. 1. There is some current debate over the absolute accuracy of these calculations and the derived conclusion that the excited $t$, states usually described by the crystal field splitting are almost entirely host-like, in contrast to the ground states which are of predominantly $d$ character. However,

C.D. (BOND.ORBITAL MODEL) $4^{\circ} \mathrm{K}$ FIVE ELECTRONS FROM $0^{\circ}$ ENTER VALFNCE BONDING

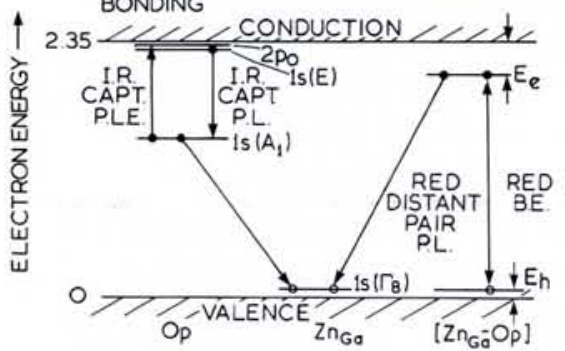

Binding Energies (Revised 1978)(meV)

$$
\begin{array}{ll}
\text { Zn Acceptor } & \text { Op Donor } \\
\text { is }\left(\Gamma_{8}\right)=70 \pm 0.5 & \text { is }\left(A_{1}\right)=898 \pm 1 \\
Z n_{G o}-O p \text { Associate } & \text { is }(E)=56.6 \pm 1 \\
E_{e}-296 \pm 6, E_{h}=44 \pm 3, & 2 p_{0}=35.9 \pm 1 \\
\text { Effective mass } E_{D}=55 \mathrm{meV}=\left(E_{D}\right)_{0} / 16.3
\end{array}
$$

Fig. 2 - Experimental properties of the onelectron state of the deep $\mathrm{O}_{\rho}$ donor and the $\mathrm{Zn} \mathrm{n}_{\mathrm{G}}$ - $O_{p}$ "molecular" isoelectronic trap in GaP. A relatively unusual feature for deep levels in semiconductors is the cross-check provided from intercomparison of energies from the several different types of optical spectra indicated. PL represents photoluminescence, PLE-PL excitation, BE-bound exciton, IR capt-infra red radiative (electron) capture.

EPS Divisions, Sections and Group

Astronomy and Astrophysics Division Solar Physics Section

Atomic and Molecular Physics Division Atomic Spectroscopy Section Chemical Physics Electronic and Atomic Collisions Molecular Physics

Computational Physics Group

Condensed Matter Division

Low Temperature Physics Section
Low Ted Matter Division

Macromolecular Physics

Magnetism

Metal Physics

Semiconductors and Insulators

Surfaces and Interfaces

High Energy \& Particle Physics Division

Nuclear Physics Division

Nuclear Physics
Optics Division

Plasma Physics Division

Quantum Electronics Division

Fig. 1 - Experimental results on the energies of the single acceptor state of the indicated cation substitutional transition metal ions in three III-V compound semiconductors. Relative to the conduction band minima, these levels indicate the threshold electronic energies $E_{e}$ required for the reaction $X^{2+}+E_{e}-X^{3+}+e_{C B}$. The energy $E_{e}$ may be estimated from thermal or optical excitation processes. The number of $3 d$ electrons in the $\mathrm{X}^{2+}$ charge states of the different transition metals is indicated at the bottom. Some individual results are still tentative; for example the level for $\mathrm{V}^{2}+$ in $\mathrm{GaAs}$ may be only $\sim 0.23 \mathrm{eV}$ below the conduction band, not approximately mid-gap as drawn. The large step between $\mathrm{Mn}^{2+}$ and $\mathrm{Fe}^{2+}$ reflects the stability due to spin stabilisation in the half filled $3 d^{5}$ shell for $\mathrm{Mn}^{2+}$ and is an important experimental effect. (Courtesy M S Skolnick)

the major chemical trends certainly seem to be well represented by this theory.

A transition between an open and closed shell behaviour may be observed across a series of semiconductors such as the $\mathrm{Zn}$ chalcogens as a function of the differential electron affinity between the $\mathrm{Cu}$ impurity and host semiconductor. Green's function and cluster calculations both suggest that even transition metal impurities like $\mathrm{Co}$ and particularly $\mathrm{Ni}$ exhibit gap states in $\mathrm{Si}$ and GaAs which are essentially dangling bondlike, with d-like states of similar symmetry deep within the valence band. However, this conclusion may be controversial. The electronic properties of some similar systems appear to exhibit strong d-character, for example from the form of phonon coupling which appears characteristic of intrad state transitions. It has been suggested recently that the $s-d$ interaction between host and transition metal may have been substantially over-emphasised, perhaps through neglect of stabilisation against covalent mixing by the strong interactions between d electrons. Neglect of many electron effects is a generally recognised weakness of the current status of deep level theory. Much more work needs to be done on the magnitudes of such effects, but this remains a daunting task.

\section{Summary}

The amount of accurate experimental data on many deep level systems has grown rapidly in the past decade. We are still far from a thorough theoretical account of the complete set of properties for any one system, however. Current indications are that each system may require particular, special treatments. Excited states are hard to calculate on the self consistent basis used for ground states. However, useful generic trends have emerged within series of closely related defects or for a given defect in different binary hosts, or as a function of ternary alloy composition. An important joint outcome of the theoretical and experimental work is the recent recognition of the importance of antisite defects in the electronic properties of semiconductors.

\section{BIBLIOGRAPHY}

For a recent general review of deep levels, see Jaros M., Deep Levels in Semiconductors (Adam Hilger, Bristol) 1982.

\section{Liquids Section}

With the approval of the Executive Committee it has been decided to set up a Liquids Section of the Condensed Matter

\section{Division.}

Individual Members of EPS interested in joining this Section should inform the Secretariat as soon as possible.

\section{ACAPPI}

Copies of the new EPS booklet of ACAPPI, Improving Interaction Between Universities and Industries, are available, free of charge, from the Secretariat. A review will be published in the next issue of Europhysics News.
Europhysics News is the official journal of the European Physical Society which comprises 29 National Socie ties, Academies and Groups, over 3000 Individual Members and 64 Associate Members. Governing bodies of EPS are the General Meeting, Council and an dies of EPS are the General Meeting, Council and an elected Executive Committee responsible for detailed policy. EPS promotes the collaboration of physicist: throughout Europe, organising and harmonising confe-
rences, and promotes international exchanges in physics including participation in research and teaching activities abroad and attendance at schools. EPS publishes in addition to EN, Europhysics Conference Abstracts, E. Ed. News and, in collaboration with The Institute of Physics (UK), the European Journal of Physics. Individual Members receive EN free of charge (price to institutions: Sw.Fr. 82.-/a), rebates on the price of many publications and on conference fees. Annual fee for Individual Members from one of the EPS member societies for 1983 is: Sw.Fr. 40.-; inde pendent members: Sw.Fr. 120. - ; APS members: Sw.Fr. 50. $-=\$ 25 .-$.

\section{Editor: E.N. Shaw}

Meetings Compilation: W.S. Newman

Editorial Board:

K. Appert, A. Baratoff, G.J. Béné,

G.R. Macleod, A. Maeder, J. Muller

Editorial and Advertising Office at the EPS Secretariat.

Address: EUROPEAN PHYSICAL SOCIETY P.O. Box 69 CH-1213 Petit-Lancy 2

Switzerland

Telephone: Geneva (22) 931130

Telex: 423455 dema ch

Cables: europhys genève

Printed by: Pfirter frères SA CH-1213 Petit-Lancy/Switzerland 